\title{
Isospin symmetry breaking and large-scale shell-model calculations with the Sakurai-Sugiura method
}

\author{
Takahiro Mizusaki ${ }^{1}$, Kazunari Kaneko ${ }^{2}$, Yang Sun ${ }^{3}$, and Shigeru Tazaki ${ }^{4}$ \\ ${ }^{1}$ Institute of Natural Sciences, Senshu University, Tokyo 101-8425, Japan \\ ${ }^{2}$ Department of Physics, Kyushu Sangyo University, Fukuoka 813-8503, Japan \\ ${ }^{3}$ Department of Physics and Astronomy, Shanghai Jiao Tong University, Shanghai 200240, People's Republic of China, Institute \\ of Modern Physics, Chinese Academy of Sciences, Lanzhou 730000, People's Republic of China \\ ${ }^{4}$ Department of Applied Physics, Fukuoka University, Fukuoka 814-0180, Japan
}

\begin{abstract}
Recently isospin symmetry breaking for mass 60-70 region has been investigated based on large-scale shell-model calculations in terms of mirror energy differences (MED), Coulomb energy differences (CED) and triplet energy differences (TED). Behind these investigations, we have encountered a subtle problem in numerical calculations for odd-odd $N=Z$ nuclei with large-scale shell-model calculations. Here we focus on how to solve this subtle problem by the Sakurai-Sugiura (SS) method, which has been recently proposed as a new diagonalization method and has been successfully applied to nuclear shell-model calculations.
\end{abstract}

The isospin symmetry breaking is one of the current topics in nuclear structure physics. An asymmetry between spectra of isospin analogue states with mirror pair nuclei has been extensively investigated in the upper $s d-$ and the lower $f p$-shell regions [1], and in mass 60-70 region [2-6]. This asymmetry, that is, isospin symmetry breaking arises partly due to the Coulomb force and partly due to the strong nucleon-nucleon interaction.

To analyze this isospin symmetry breaking, mirror energy differences (MED) [7], Coulomb energy differences (CED)[8] and triplet energy differences(TED) [7] have been discussed. The MED is a measure of charge symmetry breaking in an effective interaction and is defined as

$$
\operatorname{MED}(J)=E_{x_{T_{z}=-1}^{J, T=1}}^{J}-E_{x_{T_{z}=+1}^{J, T=1}},
$$

where $E_{x_{T}}^{J, T}$ is the excitation energies of analog states with spin $J$ and isospin $T, T_{z}$. The TED of $T=1$ states in triplet nuclei is a measure of the charge-independence breaking and is defined as

$$
T E D(J)=E_{x}^{J, T=1}+E_{T_{z}=-1}^{J, T=1}-2 E_{T_{z}=+1}^{J, T=1} .
$$

For the TED, we have to evaluate the energy of the $T=1$ and $T_{z}=0$ state for odd-odd $N=Z$ nuclei precisely, employing shell-model calculations with isospin non-conserving interactions $[5,6]$. This calculation involves a rather subtle problem in numerical calculations, especially for large-scale shell-model calculation with the widely used Lanczos method.

For the $p f$-shell or larger shells, we need largescale shell-model calculations where the $M$-scheme (shellmodel space with definite total magnetic quantum number) is often used. Third component of isospin, $T_{z}$, is also given, choosing proton and neutron numbers. As the shellmodel interaction is rotationally invariant, the obtained eigenstates naturally have a good total angular momentum. For isospin-conserving interactions, they also have a good total isospin. This property is often utilized by taking a state with good total angular momentum and isospin as an initial one of the Lanczos method.

Here we consider isospin symmetry breaking with large-scale shell-model calculations. Once the shell-model interaction contains several isospin symmetry breaking terms, total isospin is no longer a conserved quantity. This non-conservation is not, however, quantitatively significant [5] but it brings numerical difficulty for Lanczos diagonalizations, especially for odd-odd $N=Z$ nuclei with large-scale shell-model calculations.

Without isospin symmetry breaking, the low-lying states of odd-odd $N=Z$ nuclei have $T=0$ or $T=1$. As the ground state or low-spin yrast states often have $T=1$, there are many $T=0$ states below the lowest-energy $T=1$ analogue state in the $T_{z}=0$ space as shown in Fig 1. As energy increases, the level density becomes higher. In the $M$ scheme, in the case of isospin conserving interaction, $T=1$ states can be easily calculated in the $T_{z}=1$ space which has no $T=0$ states due to the isospin algebra. This calculation is, however, no longer valid for $T \approx 1$ states in the case of isospin non-conserving interactions, then we need the $T_{z}=0$ space. Here a computational problem arises. If we carry out the shell-model diagonalization with the Lanczos method in the $M$-scheme, a number of the Lanczos iteration seriously increases because there are too many $T \sim 0$ states below the analogue state. In the case of large-scale shell-model calculations, this calculation becomes very difficult or impossible to carry out. For 
this reason, a new method is clearly necessary to compute this analogue state hidden in the $T_{z}=0$ space, not in the $T_{z}=1$ space.

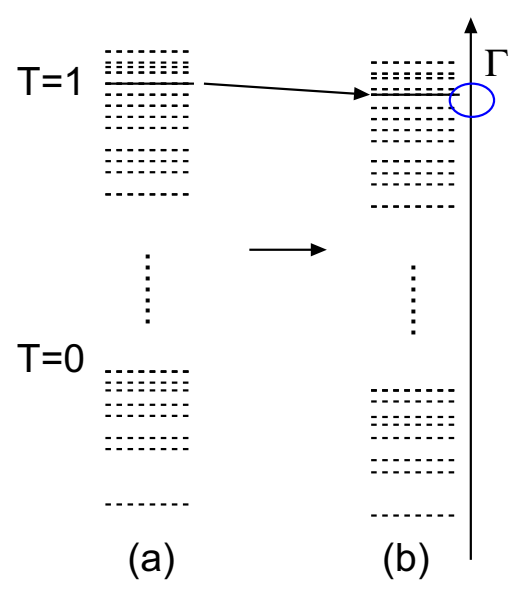

Figure 1. Schematic picture (a) Case for isospin conserving interaction: solid and dotted lines show $T=1$ and $T=0$ states, respectively. (b) Case for isospin non-conserving interaction: solid and dotted lines show $T \approx 1$ and $T \approx 0$ states, respectively.

We have proposed a new shell-model method [9] based on the Sakurai-Sugiura (SS) method [10] where largescale shell-model diagonalization can be solved by the help of the Cauchy integral on the complex plane. For this formulation, a central quantity is moments $\mu_{p}$ defined as

$$
\mu_{p}=\frac{1}{2 \pi i} \int_{\Gamma}\left\langle\phi\left|\frac{(z-\epsilon)^{p}}{z-H}\right| \phi\right\rangle d z
$$

where $p=0,1,2, \cdots$ and $H$ is a shell-model Hamiltonian. In the present calculation, as $|\phi\rangle$, we take $\left|\psi_{T=1, T_{z}=0}\right\rangle=$ $\frac{1}{N} T_{-}\left|\psi_{T=1, T_{z}=1}\right\rangle$ where $\mathcal{N}$ is a normalization factor. This state is obtained by the shell-model calculation in the $T_{z}=1$ space by switching off isospin symmetry breaking terms. $\left|\psi_{T=1, T_{z}=1}\right\rangle$ is good approximation but it is defined in the $T_{z}=1$ space, not in the $T_{z}=0$ space. By introducing an isospin lowering operator $T_{-}, T_{-}\left|\psi_{T=1, T_{z}=1}\right\rangle$ is defined in the $T_{z}=0$ space and has a good isospin $T=1$. We set $\epsilon$ by the expectation energy with $\left|\psi_{T=1, T_{z}=0}\right\rangle$ of the Hamiltonian, including isospin symmetry breaking terms. As the Cauchy integral contour $\Gamma$, we take a circle with the center $z=\epsilon$. Its radius is determined so as to include considered state as shown in Fig. 1. In this method, we can directly calculate eigenstates inside the Cauchy integral contour $\Gamma$. Moreover, the computation becomes very fast because we have well-approximated wave function.

The moments in Eq.(3) are evaluated by the numerical integration and matrix elements of the two Hankel matrices $M$ and $N$ are composed of these moments. Dimensions of these matrices are at most 100 in typical numerical calculations, independent of those of the shell-model space. Then by solving the small-scale generalized eigenvalue problem $M x=e N x$, we can obtain the energies and wave functions within the Cauchy integral contour. Note that this procedure can take full advantage of approximated wavefunction $\left|\psi_{T=1, T_{z}=0}\right\rangle$ with the SS method, unlike the Lanczos method. Its detailed procedure has been fully discussed in Refs [9, 10]. Through several applications [4-6], we confirmed that this method works well and quite efficiently. Thanks to this development with the SS method, we have overcome the subtle numerical problem in the TED, and now we have been able to calculate the TED in mass 60-70 region.

\section{References}

[1] For example, M. A. Bentley and S. M. Lenzi, Prog. Part. Nucl. Phys. 59, 497 (2007)

[2] R. Orlandi et. al., Phys. Rev. Lett. 103, 052501 (2009)

[3] L. -L. Andersson et al., Phys. Rev. C 71, 011303 (2005)

[4] K. Kaneko, S. Tazaki, T. Mizusaki, Y. Sun, M. Hasegawa, and G. de Angelis, Phys. Rev. C 82, 061301(R) (2010)

[5] K. Kaneko, T. Mizusaki, Y. Sun, S. Tazaki, and G. de Angelis, Phys. Rev. Lett. 109, 092504 (2012)

[6] K. Kaneko, Y. Sun, T. Mizusaki, and S. Tazaki, Phys. Rev. Lett. 110, 172505 (2013), Phys. Rev. C 89, 031302(R) (2014)

[7] A. P. Zuker, S. M. Lenzi, G. Martınez-Pinedo, and A. Poves, Phys. Rev. Lett. 89, 142502 (2002)

[8] S. M. Lenzi, et. al., Phys. Rev. Lett. 87, 122501 (2001)

[9] T. Mizusaki, K. Kaneko, M. Honma, and T. Sakurai, Phys. Rev. C 82, 024310 (2010), Acta Phsica Polonica B 42, 447 (2011)

[10] T. Sakurai and H. Sugiura, J. Comput. Appl. Math. 159, 119 (2003) 